\title{
Temporal attentional capture: Effects of irrelevant singletons on rapid serial visual search
}

\author{
POLLY DALTON \\ University of Oxford, Oxford, England \\ and \\ NILLI LAVIE \\ University College London, London, England
}

\begin{abstract}
The presence of a unique yet irrelevant singleton in visual search or spatial-cuing tasks is typically associated with performance costs, suggesting that singletons tend to capture attention. However, since singletons have always been spatially separated from targets in previous experiments, it remains unclear whether an irrelevant visual singleton that occurs at the same spatial location as the target but at a different point in time can produce temporal capture of attention. Here, we asked participants to search visual sequences at fixation for targets defined by size (larger or smaller than the nontargets). The presence (vs. absence) of a color singleton lengthened response times on the size discrimination task, suggesting that irrelevant singletons can lead to a temporal attentional capture.
\end{abstract}

In order to behave effectively in a complicated world, people must be able to focus their attention on goalrelevant stimuli at the expense of irrelevant ones. However, it is also important that attention can be captured by irrelevant stimuli when they are unique and may, therefore, signal potentially important changes in the environment. A central line of attention research has investigated attentional capture by such unique yet task-irrelevant singleton stimuli.

It has long been established that attentional allocation toward stimuli in nontarget locations produces performance costs, as has been shown in spatial-cuing studies (e.g., Jonides, 1980; Posner, Nissen, \& Ogden, 1978), as well as in studies of attentional capture by a singleton item presented within a visual search display (e.g., Jonides \& Yantis, 1988; Theeuwes, 1992). Both these areas of research address the consequences of paying attention to irrelevant spatial locations. More recently, research has begun to address the effects of attentional allocation to irrelevant temporal positions. However, very little previous research has investigated the possibility of attentional capture by stimuli appearing at irrelevant temporal positions. Here, we ask whether a unique yet irrelevant singleton can produce temporal attentional capture during a rapid serial visual presentation (RSVP) search task.

This research was supported by an Engineering and Physical Sciences Research Council (U.K.) Studentship, Economic and Social Sciences Research Council (U.K.) Postdoctoral Fellowship PTA-026-27-0550, and a Junior Research Fellowship from St Anne's College, Oxford, to P.D. We thank Chip Folk, Andy Leber, and Jan Theeuwes for their helpful comments on an earlier draft of this work. Correspondence concerning this article should be addressed to P. Dalton, Department of Psychology, Royal Holloway University of London, Egham, Surrey TW20 0EX, England (e-mail: polly.dalton@rhul.ac.uk).
A few recent studies have provided some evidence that attentional allocation to items presented at irrelevant temporal positions can lead to performance costs. For example, Folk, Leber, and Egeth (2002) have shown attentional capture in an RSVP task by color singleton distractors flanking the central RSVP letters. However, since the singleton distractors in this study were spatially separated from the targets, it is not clear whether the capture effects were due to diversion of attention to an irrelevant spatial location (i.e., spatial attentional capture) or to an irrelevant temporal position (i.e., temporal attentional capture), or both.

Research into the attentional blink (AB) has shown that attending to (rather than ignoring) the first of two targets can prevent participants from detecting the second, as long as the second target occurs within $500 \mathrm{msec}$ of the first (e.g., Broadbent \& Broadbent, 1987; Raymond, Shapiro, \& Arnell, 1992). Thus, attentional allocation toward one item in a serial stream can interfere with processing of an item at a different point in the stream. These results cannot be explained in terms of spatial attention, because all the items were presented in a central RSVP stream. However, since participants respond primarily to the first target and only later to the second target, the $\mathrm{AB}$ is likely to involve both response- and memory-related effects (e.g., Jolicœur, 1998). Moreover, most of the AB research assesses the consequences of attending deliberately to target stimuli and, as such, does not provide information about the involuntary capture of attention.

Recent findings from the $\mathrm{AB}$ paradigm that an ignored first target or additional singleton (e.g., a colored box around a nontarget letter) can interfere with recall of the second target are more informative about the possibility of involuntary temporal attentional capture (e.g., Chun, 1997; Folk, Leber, \& Egeth, 2001; Maki \& Mebane, 2006; 
Wee \& Chua, 2004). However, in all these studies, reliable performance costs were produced only by items that were defined on the same dimension as the targets (e.g., they were both color singletons; see also Ghorashi, Zuvic, Visser, \& Di Lollo, 2003). ${ }^{1}$ Therefore, these results cannot speak to the possibility of involuntary temporal attentional capture by a singleton distractor defined on a task-irrelevant dimension.

We have recently demonstrated temporal attentional capture by such task-irrelevant auditory singletons during sequential auditory search (Dalton \& Lavie, 2004). Irrelevant auditory singleton distractors (i.e., sounds that were unique on one dimension-e.g., frequency) captured attention even though the targets were defined on a different dimension (e.g., intensity). However, whereas hearing tends to prioritize temporal information, vision operates more on spatial coordinates, and it is, therefore, unclear whether analogous temporal visual attentional capture effects can be found.

We used a visual search attentional capture task, in which unique singleton items typically attract attention despite being irrelevant to the task. However, we modified the task to present both target and nontarget stimuli in a sequential stream, rather than in a spatial array. Any evidence of a cost due to singletons presented at a different temporal position from the target but at the same spatial location would suggest pure temporal capture of attention.

\section{EXPERIMENT 1}

In this experiment, we asked whether an irrelevant color singleton would capture attention during an RSVP search task. The participants searched visual letter sequences for targets that were either larger or smaller than the rest of the letters. On $50 \%$ of the trials, an irrelevant color singleton was presented (e.g., a red letter among black). Any cost to target detection in the presence (vs. absence) of the singleton would be suggestive of attentional capture.

\section{Method}

\section{Participants}

The participants in all the experiments were paid students (18-35 years of age). All reported normal or corrected-to-normal vision. Eight participants took part in this experiment.

\section{Stimuli and Procedure}

The experiments were created and run on a PC using E-Prime (Psychology Software Tools, Pittsburgh). Each sequence started with a black fixation cross presented at the center of the screen for $500 \mathrm{msec}$, followed by a $50-\mathrm{msec}$ blank screen. A sequence of five uppercase letter Ns was then presented, one after another at the center of the screen. Each appeared for $60 \mathrm{msec}$, followed by a 70 -msec blank screen. At a viewing distance of $60 \mathrm{~cm}$, nontargets and singletons subtended a visual angle of $1.4^{\circ} \times 1.4^{\circ}$, large targets subtended $1.7^{\circ} \times 1.7^{\circ}$, and small targets subtended $1.1^{\circ} \times 1.1^{\circ}$. The participants were requested to respond with a keypress: 1 for large target or 2 for small target, using the index and middle fingers of the right hand, respectively, upon presentation of a question mark at the center of the screen at the end of each sequence. A feedback screen displaying the words correct (in blue), incorrect (in red) or no response detected (in red) was presented at the end of each trial, either after a response had been collected or after 3,000 msec if no response had been made.

Targets appeared on every trial and were equally likely to be large or small and in the third or fourth position. Irrelevant distractor singletons appeared on $50 \%$ of the trials, directly before or after the target with equal probability. All the letters were presented against a white background. The nontargets and targets were black for half the participants (red for the other half), and the singletons were red for these participants (black for the other half). The participants were asked to focus on letter size and to ignore any variation in other dimensions. They were informed that some distractor items of a different color would occur and were warned that their performance might be harmed if they failed to ignore these distractors. A short practice block of 16 trials preceded two experimental blocks, each containing 80 trials.

\section{Results}

A preliminary analysis of all three experiments indicated that the between-subjects factor of target color (red vs. black) did not interact with the factor of singleton presence (vs. absence) ( $p>.30$ for all comparisons). All the data are thus pooled across target color. In all the experiments, incorrect responses were excluded from the response time (RT) analysis, as were RTs longer than 1,500 msec. Table 1 presents mean RTs and error rates across participants as a function of singleton presence (present vs. absent) and target type (large vs. small).

\section{Response Times}

A two-way within-subjects ANOVA using these factors revealed a significant main effect of singleton presence $\left[F(1,7)=7.40, M S_{\mathrm{e}}=14,573.09, p<.05\right]$. Target RTs were longer on singleton-present trials $(M=426 \mathrm{msec})$ than on singleton-absent trials $(M=384 \mathrm{msec})$, suggesting that the color singleton captured attention despite being irrelevant to the task. There was also a main effect of target type, indicating that responses were faster when the target was large $(M=377 \mathrm{msec})$ than when it was small $(M=433 \mathrm{msec})\left[F(1,7)=7.64, M S_{\mathrm{e}}=25,254.60, p<\right.$ $.05]$. This is in line with previous research demonstrating an advantage for large targets among small nontargets, in comparison with small targets among large nontargets, in spatial visual search (e.g., Treisman \& Gormican, 1988). The factors of singleton presence and target type did not interact significantly $\left[F(1,7)=1.98, M S_{\mathrm{e}}=1,979.31\right.$, $p=.20]$.

Table 1 Averages of Participants' Mean Response Times (RTs, in Milliseconds, With Standard Errors) and Mean Error Rates (\%E) for Experiment 1 as a Function of Singleton Presence and Target Type

\begin{tabular}{|c|c|c|c|c|c|c|c|c|}
\hline \multirow[b]{4}{*}{ Target } & \multicolumn{6}{|c|}{ Singleton } & & \\
\hline & \multicolumn{3}{|c|}{ Absent (A) } & \multicolumn{3}{|c|}{ Present $(\mathrm{P})$} & \multirow{2}{*}{\multicolumn{2}{|c|}{$\begin{array}{c}\text { Interference } \\
(\mathrm{P}-\mathrm{A})\end{array}$}} \\
\hline & \multicolumn{2}{|c|}{ RT } & \multirow[b]{2}{*}{$\% \mathrm{E}$} & \multicolumn{2}{|c|}{ RT } & \multirow[b]{2}{*}{$\% \mathrm{E}$} & & \\
\hline & $M$ & SE & & $M$ & SE & & RT & $\%$ \\
\hline Large & 363 & 30 & 7 & 390 & 37 & 8 & 27 & 1 \\
\hline Small & 404 & 48 & 10 & 462 & 45 & 11 & 58 & 1 \\
\hline
\end{tabular}


A further one-way within-subjects ANOVA on the data from singleton-present trials revealed a significant effect of singleton position (before vs. after the target), so that responses were slower when the singleton occurred before $(M=449 \mathrm{msec})$ versus after $(M=400 \mathrm{msec})$ the target $\left[F(1,7)=9.01, M S_{\mathrm{e}}=9,381.38, p<.05\right]$. Indeed, RTs when the singleton occurred after the target were not significantly different from RTs when the singleton was absent $[M=383 \mathrm{msec} ; t(7)=1.14, p=.29]$. Thus, the appearance of a singleton before the target was more damaging to target processing than was the appearance of a singleton after the target. This may be due to some target processing occurring without competition when the singleton occurs after the target.

Finally, to confirm that capture could be found from singletons at entirely irrelevant temporal positions, we looked separately at the effects of singleton presence (absent vs. present) for singletons in Serial Position 2 (where the target never occurred) and singletons in Serial Position 3 (where the target could occur). The finding of a significant capture effect by singletons at Position $2[M$ effect $=56 \mathrm{msec} ; t(7)=2.44, p<.05]$ that was not significantly different $(F<1)$ from the effects of singletons at the potential Target Position $3[M$ effect $=78 \mathrm{msec}$; $t(7)=2.32, p=.05]$ indicates that the capture effects were not restricted to singletons occurring in potential target positions and, thus, cannot be attributed to voluntary allocation of attention to those positions. We note, nevertheless, that the nonsignificant numerical trend might suggest that voluntary attentional allocation can increase the magnitude of singleton interference.

\section{Errors}

The two-way error ANOVA with the factors of singleton presence and target type revealed no significant main effects or interactions ( $p>.20$ for all comparisons). Note, however, that the error rates showed trends similar to those for the RTs (see Table 1).

A further one-way within-subjects ANOVA on error data from singleton-present trials, using the factor of singleton position (before vs. after the target), revealed a trend for a higher error rate when the singleton occurred before $(M=$ $11 \%)$ versus after $(\mathrm{M}=8 \%)$ the target $[F(1,7)=4.94$, $\left.M S_{\mathrm{e}}=36.00, p=.062\right]$. Error rates in the latter condition were very similar to error rates when the singleton was absent $(M=8.5 \%)$. Thus, as for the RTs, the singleton appeared to cause more interference when it appeared before, rather than after, the target.

In conclusion, the present experiment showed significant interference due to the presence of an irrelevant color singleton in an RSVP discrimination task. This finding is suggestive of attentional capture by the irrelevant singleton.

\section{EXPERIMENT 2}

We have argued that the interference found in Experiment 1 is likely to have been due to attentional capture by the irrelevant color singleton. However, since the single- ton in Experiment 1 always appeared either directly before or directly after the target, it is possible that this interference might have been a result of contrast effects, so that it might be easier to judge target size in comparison with a nontarget of the same color than with a nontarget (singleton) of a different color. Experiments $2 \mathrm{~A}$ and $2 \mathrm{~B}$ were designed to examine this alternative account.

The participants in Experiments $2 \mathrm{~A}$ and $2 \mathrm{~B}$ carried out the size discrimination task used in Experiment 1. As in Experiment 1, all the stimuli were in the same color on $50 \%$ of the trials; but unlike in Experiment 1, the stimuli alternated between the target color and a distractor color on the remaining $50 \%$ of the trials. On these color alternation trials, the target was presented in between two items of a different color, and if the interference effects seen in Experiment 1 were due to contrast effects, they should therefore persist in the alternation condition. By contrast, if the interference effects were due to attentional capture by the presence of a unique color singleton, they should be eliminated in the alternation condition. Experiment $2 \mathrm{~A}$ used sequences of six or seven letters, whereas Experiment $2 \mathrm{~B}$ used sequences of four or five letters (for reasons described below).

\section{Method}

\section{Participants}

Eight new participants took part in Experiment 2A, and a further 8 in Experiment 2B.

\section{Stimuli and Procedure}

The equipment and stimuli were the same as those described in Experiment 1. Targets and nontargets were black for half the participants (red for the other half), and the distractors were red for these participants (black for the other half). On $50 \%$ of the trials, sequences were made up only of nontargets and targets, so that each sequence was presented in the target color only. On the remaining $50 \%$ of the trials, the letters alternated in color between the target color and the irrelevant distractor color. Alternating sequences were just as likely to start with the distractor color as with the target color. In order to avoid any contingency between the color of the first letter in the sequence and subsequent target position, the sequences consisted of either six or seven letters, with equal probability, in Experiment 2A. Similarly, the sequences in Experiment 2B consisted of either four or five letters, with equal probability. The targets were equally likely to be large or small. In Experiment 2A, they appeared on every trial in the fourth or fifth position of the sixletter sequences and in the fifth or sixth position of the seven-letter sequences. In Experiment 2B, they appeared on every trial in the second or third position of the four-letter sequences and in the third or fourth position of the five-letter sequences. All other aspects were the same as in Experiment 1.

\section{Results and Discussion}

Table 2 presents mean RTs and error rates for Experiments $2 \mathrm{~A}$ and $2 \mathrm{~B}$ as a function of alternation condition (alternation absent vs. present) and target type (large vs. small).

\section{Experiment 2A}

RTs. A two-way within-subjects ANOVA using these factors showed no significant main effects or interactions ( $p>.20$ for all comparisons). It is especially impor- 
Table 2

Averages of Participants' Mean Response Times (RTs, in Milliseconds, With Standard Errors) and Mean Error Rates (\%E) for Experiments $2 \mathrm{~A}$ and $2 B$ as a Function of Alternation Condition and Target Type

\begin{tabular}{|c|c|c|c|c|c|c|c|c|c|}
\hline & \multirow[b]{4}{*}{ Target } & \multicolumn{6}{|c|}{ Alternation Condition } & & \\
\hline & & \multirow{2}{*}{\multicolumn{2}{|c|}{$\begin{array}{l}\text { Absent } \\
\text { RT }\end{array}$}} & \multirow[b]{3}{*}{$\% \mathrm{E}$} & \multicolumn{3}{|c|}{ Present $(\mathrm{P})$} & \multirow{2}{*}{\multicolumn{2}{|c|}{$\begin{array}{l}\text { Interference } \\
\qquad(\mathrm{P}-\mathrm{A})\end{array}$}} \\
\hline & & & & & \multicolumn{2}{|c|}{ RT } & \multirow[b]{2}{*}{$\% \mathrm{E}$} & & \\
\hline & & $M$ & $\overline{S E}$ & & $M$ & $\overline{S E}$ & & RT & $\%$ \\
\hline \multirow[t]{2}{*}{ Experiment 2A } & Large & 282 & 27 & 3 & 278 & 29 & 1 & -4 & -2 \\
\hline & Small & 298 & 28 & 2 & 306 & 41 & 2 & 8 & 0 \\
\hline \multirow[t]{2}{*}{ Experiment 2B } & Large & 337 & 49 & 8 & 316 & 42 & 7 & -21 & -1 \\
\hline & Small & 339 & 49 & 11 & 346 & 47 & 9 & 7 & -2 \\
\hline
\end{tabular}

tant that there was no main effect of the presence $(M=$ $290 \mathrm{msec})$ versus absence $(M=292 \mathrm{msec})$ of the color alternation $(F<1)$.

A between-experiment ANOVA confirmed that the singleton effect in Experiment $1(M=42 \mathrm{msec})$ was significantly larger than the null color alternation effect in Experiment $2 \mathrm{~A}(M=-2 \mathrm{msec})\left[F(1,14)=5.17, M S_{\mathrm{e}}=\right.$ $3,211.61, p=.039]$. Thus, the singleton interference effect in Experiment 1 cannot be explained in terms of simple color contrast effects.

The between-experiment ANOVA also found a significant main effect of experiment, so that RTs were shorter in the present experiment $(M=286 \mathrm{msec})$ than in Experiment $1(M=402 \mathrm{msec})[F(1,14)=10.12, p<.01]$. As can be seen in Tables 1 and 2, this effect was found in the absence, as well as the presence, of the singleton or color alternation. This may be because the present experiment used stimulus sequences of six or seven items, whereas Experiment 1 used sequences of five items. Although the additional items in the present experiment were presented at the beginning of the sequences (so that the time in between the appearance of the target and the response window was the same in both experiments), it is possible that the longer sequences used here allowed the participants to prepare more effectively for the subsequent target presentation, leading to shorter target RTs. For this reason, Experiment $2 \mathrm{~B}$ used shorter sequences, with the aim of reducing performance to a level comparable to that in Experiment 1.

Errors. A two-way within-subjects ANOVA with the factors of alternation condition and target type showed no main effect of either factor ( $p>.20$ for both comparisons). The interaction between alternation presence and target type was not significant $\left[F(1,7)=3.38, M S_{\mathrm{e}}=\right.$ 9.03, $p=.11]$. Note that any trend toward a higher error rate for large targets in the absence (vs. presence) of the alternation (see Table 2) is in the direction opposite to that of the singleton interference effect, and in any case, this trend was not significant $[t(7)=1.70, p=.13]$.

\section{Experiment 2B}

RTs. A two-way within-subjects ANOVA revealed no significant main effects or interactions $(p>.09$ for all comparisons). As in the previous experiments, there was a trend for faster responses to large targets than to small targets $\left[F(1,7)=3.61, M S_{\mathrm{e}}=2,070.14, p=.10\right]$. Criti- cally, once again, there was no effect for the presence $(M=331 \mathrm{msec})$ versus absence $(M=338 \mathrm{msec})$ of color alternation $(F<1)$.

A between-experiment ANOVA confirmed that the singleton effect found in Experiment $1(M=42 \mathrm{msec})$ was significantly larger than the null effect of color alternation in Experiment $2 \mathrm{~B}(M=-7 \mathrm{msec})[F(1,14)=$ $\left.7.35, M S_{\mathrm{e}}=547.72, p=.017\right]$. Thus, the present experiment replicated the results of Experiment 2A, suggesting that the singleton interference effects observed in Experiment 1 were due to the presence of a single unique item and cannot be explained in terms of lower level contrast effects. Importantly, unlike in Experiment 2A, there was no systematic difference in RTs between Experiment $1(M=$ $402 \mathrm{msec})$ and the present experiment $(M=334 \mathrm{msec})$ $\left[F(1,14)=2.7, M S_{\mathrm{e}}=26,874.79, p=.12\right] .{ }^{2}$ In fact, the nonsignificant numerical trend is an overestimation, due to the inclusion of trials in which the singleton/alternation is present (since these trials elevated the RTs in Experiment 1, due to the singleton cost, but did not do so in Experiment 2B). Removal of these trials gives overall mean RTs of $384 \mathrm{msec}$ for Experiment 1 and $338 \mathrm{msec}$ for Experiment $2 \mathrm{~B}$, which are not significantly different from each other $[t(14)=1.25, p=.23]$.

Errors. A two-way within-subjects error ANOVA with the factors of alternation condition and target type showed no main effect of alternation condition and no interaction between alternation condition and target type $(F<1$ for both comparisons). There was a trend toward a main effect of target type $\left[F(1,7)=4.20, M S_{\mathrm{e}}=11.32, p=.08\right]$, suggesting, as in the previous experiments, that the participants made more errors when the target was small $(M=$ $9.9 \%)$ than when it was large $(M=7.5 \%)$. Importantly, error rates in the present experiment $(M=8.7 \%)$ did not differ significantly from error rates in Experiment 1 $(M=8.9 \%)$, indicating, in line with the RT results, that the shorter sequences used here did, in fact, reduce performance to a level comparable to that in Experiment 1.

Overall, Experiments 2A and 2B have shown that color alternation in the visual search sequences used here does not produce reliable interference. This suggests that the interference effect found in Experiment 1 is likely to have been due to the presence of a unique color singleton, rather than simply to lower level factors associated with color contrast effects. 


\section{GENERAL DISCUSSION}

The present study provides the first demonstration of pure temporal attentional capture by singletons defined on a task-irrelevant dimension. This capture effect critically depended on the distractor's being a singleton and could not be attributed to color contrast effects (Experiment 2).

In all previous examinations of potential capture of attention in temporal search, performance costs were produced by singletons that served as targets (producing an AB; e.g., Raymond et al., 1992) or were presented in a different spatial position (e.g., Folk et al., 2002; Wee \& Chua, 2004) or were defined on the target dimension (e.g., Chun, 1997; Folk et al., 2001; Maki \& Mebane, 2006). The present results, therefore, provide the first demonstrations of pure temporal attentional capture by singletons defined on a task-irrelevant dimension. Moreover, capture effects here generalized to singletons in unattended serial positions (where the target could never appear), and those positions should not have been attended voluntarily, since singletons did not serve as valid cues for target position (occurring before targets on only $25 \%$ of the trials). Thus, the present results are likely to reflect capture of attention, rather than voluntary attentional allocation toward the singleton.

Nevertheless it is possible that the capture we have found might be open to top-down influences. For example, the participants in the present experiments may have adopted a singleton detection strategy (Bacon \& Egeth, 1994), searching for any singleton item, meaning that both color (nontarget) and size (target) singletons would have been prioritized. This possibility is currently under investigation in our lab.

Overall, although many previous studies have demonstrated that irrelevant singletons can capture visual attention if they appear as part of a spatial array, here we have clearly established such singleton capture of attention in the temporal domain. These results thus strengthen previous claims that the visual system is tuned to detecting items that are unique against the background stimulation yet irrelevant to an ongoing task.

\section{REFERENCES}

BaCon, W. F., \& EgEth, H. E. (1994). Overriding stimulus-driven attentional capture. Perception \& Psychophysics, 55, 485-496.

Broadbent, D. E., \& Broadbent, M. H. P. (1987). From detection to identification: Response to multiple targets in rapid serial visual presentation. Perception \& Psychophysics, 42, 105-113.
Chun, M. M. (1997). Temporal binding errors are redistributed by the attentional blink. Perception \& Psychophysics, 59, 1191-1199.

Dalton, P., \& Lavie, N. (2004). Auditory attentional capture: Effects of singleton distractor sounds. Journal of Experimental Psychology: Human Perception \& Performance, 30, 180-193.

Folk, C. L., Leber, A. B., \& Egeth, H. E. (2001, November). Capture at the fovea: Control settings and the attentional blink. Paper presented at the 42nd Annual Meeting of the Psychonomic Society, Orlando, FL.

FolK, C. L., Leber, A. B., \& Egeth, H. E. (2002). Made you blink! Contingent attentional capture produces a spatial blink. Perception \& Psychophysics, 64, 741-753.

Ghorashi, S. M. S., Zuvic, S. M., Visser, T. A.W., \& Di Lollo, V. (2003). Focal distraction: Spatial shifts of attentional focus are not required for contingent capture. Journal of Experimental Psychology: Human Perception \& Performance, 29, 78-91.

Joliceur, P. (1998). Modulation of the attentional blink by on-line response selection: Evidence from speeded and unspeeded Task deci- $^{-}$ sions. Memory \& Cognition, 26, 1014-1032.

Jonides, J. (1980). Towards a model of the mind's eye's movement. Canadian Journal of Psychology, 34, 103-112.

Jonides, J., \& YANTIS, S. (1988). Uniqueness of abrupt visual onset in capturing attention. Perception \& Psychophysics, 43, 346-354.

MaKi, W. S., \& Mebane, M. W. (2006). Attentional capture triggers an attentional blink. Psychonomic Bulletin \& Review, 13, 125-131.

Posner, M. I., Nissen, M. J., \& Ogden, W. C. (1978). Attended and unattended processing modes: The role of set for spatial location. In H. L. Pick, Jr. \& E. Saltzman (Eds.), Modes of perceiving and processing information (pp. 160-174). Hillsdale, NJ: Erlbaum.

RaYmond, J. E., Shapiro, K. L., \& Arnell, K. M. (1992). Temporary suppression of visual processing in an RSVP task: An attentional blink? Journal of Experimental Psychology: Human Perception \& Performance, 18, 849-860.

Theeuwes, J. (1992). Perceptual selectivity for color and form. Perception \& Psychophysics, 51, 599-606.

Treisman, A., \& Gormican, S. (1988). Feature analysis in early vision: Evidence from search asymmetries. Psychological Review, 95, 1548.

Wee, S., \& ChuA, F. K. (2004). Capturing attention when attention "blinks." Journal of Experimental Psychology: Human Perception \& Performance, 30, 598-612.

\section{NOTES}

1. An irrelevant singleton (an abruptly onset shape in search for color targets) was presented in one of these experiments (Wee \& Chua, 2004, Experiment 1). However, this did not produce clear capture effects. By contrast, the effects of singletons that shared the target feature or were presented at a different spatial location from the rest of the stream were clear and reliable.

2. We note that there was no difference $(F=1)$ in overall mean RTs between Experiments 1 and $2 \mathrm{~B}$, even when the analysis is restricted to the five-letter trials from Experiment 2B (which are directly comparable to those in Experiment 1; $M$ from five-letter trials in Experiment $2 \mathrm{~B}=$ $324 \mathrm{msec}$ ).

(Manuscript received June 24, 2005; revision accepted for publication January 30, 2006.) 